\title{
Telomerase suppression initiates PML-dependent p53 activation to inhibit bladder cancer cell growth
}

\author{
YAN XUE, LEI LI, DONG ZHANG, KAIJIE WU, PENG GUO, JIN ZENG, XINYANG WANG and DALIN HE \\ Department of Urology, the First Affiliated Hospital of Medical School, Xi'an Jiaotong University, \\ Xi'an 710061, Shaanxi, P.R. China
}

Received July 28, 2010; Accepted August 31, 2010

DOI: $10.3892 /$ or_00001017

\begin{abstract}
Most human cancer cells maintain telomere to immortalization through telomerase activity. Inhibition of telomerase activity is a powerful strategy for cancer therapy; however, the potential molecular signals following telomerase suppression are still not clear. Promyelocytic leukemia protein (PML) is an essential component of PML nuclear bodies and a tumor suppressor in bladder cancer. In this study, using mutant human telomerase reverse transcriptase (hTERT) or shRNA to inhibit telomerase activity, we found telomerase suppression increased the expression of PML and resulted in its translocation to the nucleus in bladder cancer T24 cells. Additionally, we found that p53 was recruited into nucleus and colocalized with PML after telomerase suppression. Subsequently, there was a decrease in cell growth in vitro and in vivo and an increase in cell cycle arrest and apoptosis. Furthermore, we showed here that PML is indispensable for p53 nuclear translocation and p21 induction after telomerase inhibition. Therefore, our data indicate that suppression of telomerase could activate the PML-dependent p53 signaling pathway and inhibit bladder cancer cell growth, and also provide new insight into the potential crosstalk between PML and hTERT in bladder cancer cells.
\end{abstract}

\section{Introduction}

Telomere is a special and highly conserved structure at the ends of eukaryotic chromosomes and its stabilization is necessary for tumor immortalization (1). Most of human tumors including bladder cancer maintain infinite replicative potential through the activation of telomerase (2). Telomerase is a large ribonucleoprotein composed of an RNA subunit (hTR), telomerase-associated protein 1 (TP1), and the human catalyst telomerase reverse transcriptase (hTERT) (3). hTERT is concomitantly expressed with the activation

Correspondence to: Dr Dalin He, Department of Urology, the First Affiliated Hospital of Medical School, Xi'an Jiaotong University, Xi'an 710061, P.R. China

E-mail:dalinhexjtu@126.com

Key words: telomerase, telomerase reverse transcriptase, promyelocytic leukemia protein, $\mathrm{p} 53$, bladder cancer of telomerase during cellular immortalization and tumor progression. Introduction of hTERT into telomerase negative cells leads to telomerase activation (4), while inhibition of hTERT impairs telomerase activity in various tumor cells (5). Thus, hTERT is a rate limiting factor for telomerase activity and plays a key role in telomerase cellular function. Meanwhile, accumulated evidence showed that telomerase had cancer-prompting properties in mediating cellular proliferation, survival, and tumorigenesis, which were independent of its role in telomere maintenance (6).

The gene encoding the promyelocytic leukemia protein (pml) was first identified as PML-RARa and RARa-PML fusion protein as a result of the $\mathrm{t}(15 ; 17)$ chromosomal translocation in acute promyelocytic leukemia (7). PML is localized in the discrete speckle nuclear structures known as the PML nuclear bodies (PML-NBs) or PML oncogenic domains (PODs) (8). These nuclear bodies are critical for PML physical function and recruit many cellular components, such as Sp100, p53, Daxx and CBP300 (9). PML is a multifunctional protein that plays essential roles in regulating critical cellular functions, including replicative senescence, cellular proliferation, apoptosis, and DNAdamage response (8). Recent studies have identified PML as a tumor suppressor (8). Our previous studies have demonstrated that PML expression has a negative correlation with the stage and grade of bladder cancer, and overexpression of PML could inhibit the growth of bladder cancer cells and induce cell apoptosis via a caspase-dependent pathway $(10,11)$.

Several strategies have been developed to inhibit the telomerase activity as a novel cancer specific therapy. In addition, more and more cellular proteins regulating telomerase activity have been identified, such as p53, RB, Myc and WT1 (12). However, the relationship between telomerase and PML has not been fully investigated. In the present study, using a dominant negative mutant vector of hTERT or specific shRNA to inhibit telomerase activity in telomerase positive bladder cancer T24 cells, we provide the evidence that telomerase suppression was able to activate PML-dependent p53 signaling pathway and inhibit bladder cancer cell growth in vitro and in vivo.

\section{Materials and methods}

Cell culture and reagents. The human bladder cancer cell line T24 was cultured in Dulbecco's modified Eagle's 
Medium (Gibco, Grand Island, NY) supplemented with $10 \%$ (v/v) heat-inactivated fetal bovine serum (FBS, Sijiqing, Hangzhou, China) at $37^{\circ} \mathrm{C}$ with $5 \% \mathrm{CO}_{2}$ in a humidified incubator. Antibodies against PML, p53 and p21 were purchased from Santa Cruz Biotechnology Inc. (Santa Cruz, CA).

Cell transfection and establishment of stable transfectants. Dominant negative (DN) mutant reconstruct (PCIneo/hTERTDN868A), short hairpin RNA (shRNA) of hTERT and PML siRNA were transfected into cells using Lipofectamine 2000 (Invitrogen, Gaithersburg, VA) according to manufacturer's protocol, with empty vector, negative shRNA or siRNA as control, respectively. ShRNA of TERT and siRNA targeting PML were designed and synthesized by Invitrogen (Carlsbad, CA, USA). The sequence of shRNA targeting hTERT was CACGCGTCGT GGAGACCATCTTTCTTTCAAGAGAAGAAAGATGG TCTCCACGAGCTTTTTTG (sense); GATCCAAAAAA GCTCGTGGAGACCATCTTTCTTCTCTTGAAAGAAAG ATGTCTCCACGAGC (antisense). The oligo sequence for PML was GGAAGACUCAGAUGCCGAATT (sense); UUCGGCAUCUGAGUCUUCCTT (antisense). Stable hTERT dominant negative mutant transfectants were selected with $300 \mu \mathrm{g} / \mathrm{ml} \mathrm{G} 418$ and verified by a telomerase activity assay and designated as T24/DN868A and T24/PCI.

TRAP-PCR-ELISA telomerase activity assay. Telomerase activity was measured using a TRAP-PCR-ELISA assay kit following the manufacturer's instructions (Roche, Basel, Switzerland). Briefly, $2 \times 10^{5}$ cells were suspended with lysis buffer, and an equal amount of nuclear supernatant was used as a TRAP template for PCR reaction. The reaction mixture was incubated at $25^{\circ} \mathrm{C}$ for $30 \mathrm{~min}$, and then PCR was amplified for 35 cycles at $94^{\circ} \mathrm{C}$ for $30 \mathrm{sec}$ and $59^{\circ} \mathrm{C}$ for $60 \mathrm{sec}$. Then product was mixed with a hybridization solution and incubated at $37^{\circ} \mathrm{C}$ for $1 \mathrm{~h}$, followed by washing and incubating for $30 \mathrm{~min}$. It was then chromogenized and the absorbance was detected. For each assay, three independent experiments were performed.

Q-FISH for cytogenetic analysis. Chromosomes from colcemid arrest cells were prepared and followed a standard Q-FISH technique (13) using the Telomere PNA FISH/FITC kit (Dako Cytomation, USA). In brief, detached cells were treated with $50 \mathrm{mmol} / \mathrm{K} \mathrm{KCl}$ and fixed using 3:1 methanol/ glacial acetic acid; then slides were prepared. Telomere was detected using an FITC-conjugated peptide nucleic acid (PNA) probe and counterstained with DAPI. Digital images were captured under confocal microscopy at x600 magnification.

Immunofluorescence. Cells were grown on glass coverslips and fixed in $4 \%$ paraformaldehyde (PFA). After washing with PBS, cells were permeabilized using $0.1 \%$ Triton X-100 solution and blocked with $10 \%$ donkey serum. Following incubation with the primary anti-PML and anti-p53 antibodies at $4^{\circ} \mathrm{C}$ overnight, cells were washed and incubated with TRITC-goat anti mouse and FITC-goat anti rabbit secondary antibodies for $30 \mathrm{~min}$ at room temperature. After stained with DAPI for 10 min, images were collected under confocal microscopy.

Methyl thiazolyl tetrazolium (MTT) assay. Cell growth rate was determined by MTT proliferation assay as in our previous study (14). In brief, various transfectants were seeded in 96well plates, and $20 \mu \mathrm{l}$ MTT (5 mg/ml MTT in PBS, Sigma, St. Louis, MO) was added at indicated times. After 4-h incubation at $37^{\circ} \mathrm{C}$ in $5 \% \mathrm{CO}_{2}$, the culture medium containing MTT was removed and $150 \mu 1 \mathrm{DMSO}$ was added to solubilize the formazan crystals. The absorbance (O.D.) at $590 \mathrm{~nm}$ was measured using the Microplate Autoreader (Bio-Tek Instruments, Vermont, USA). For each assay, three independent experiments were performed.

Flow cytometry. Cells were harvested and washed by PBS. For cell cycle assay, cells were fixed with $70 \% \mathrm{EtOH}$, and stained with propidium iodide (PI, $50 \mu \mathrm{g} / \mathrm{ml}$ ) in the presence of RNaseA $(10 \mu \mathrm{g} / \mathrm{ml})$. Annexin V-FITC and PI staining using an apoptosis kit (Invitrogen) were performed to detect cellular apoptosis. Mitochondrial transmembrane potential $\left(\Delta \Psi_{\mathrm{m}}\right)$ were determined by using JC-1 probes. Stained cells were examined by flow cytometric analysis on a FACS Calibur (Becton-Dickinson \& Co., San Jose, CA). For each assay, three independent experiments were performed.

Tumorigenicity assay in vivo. Four to six weeks old nude mice (Shanghai Experimental Animal Center, Shanghai, China) were used for the tumorigenicity assay. T24, T24/ PCI and T24/DN868A cells were harvested, washed, and resuspended in serum-free DMEM, and $1 \times 10^{6}$ cells were injected subcutaneously. The mice were sacrificed after 8 weeks to measure the tumor size. H\&E and immunohistochemical staining was carried out as described previously (15). All experiments on the animals complied with the Guidelines of Animal Care of Xi'an Jiaotong University.

Western blotting. Extraction of cytosolic and nuclear extracts and Western blotting were carried out as described previously $(11,16)$. Briefly, samples were analyzed by $12 \%$ SDS-PAGE, transferred to nitrocellulose membranes, and probed with the primary antibodies and secondary antibodies coupled to horseradish peroxidase, then detected by the ECL chemiluminescent detection system (Amersham, Piscataway, $\mathrm{NJ}$ ).

Statistical analysis. All data analysis was done by the software SPSS 13.0 for Windows. $\mathrm{P}<0.05$ was regarded as the threshold value for statistical significance.

\section{Results}

Telomerase activity and telomere length decreased in T24/DN868A and T24/shTERT cells. To constitutively inactivate hTERT, human bladder cancer cell line T24 with high telomerase activity was transfected with a plasmid encoding a dominant-negative (DN) hTERT, and individual cell clones were isolated by G418 screening for 7 weeks. TRAP-PCR-ELISA analysis demonstrated that in T24/ DN868A cells, telomerase activity was sharply decreased to 
A

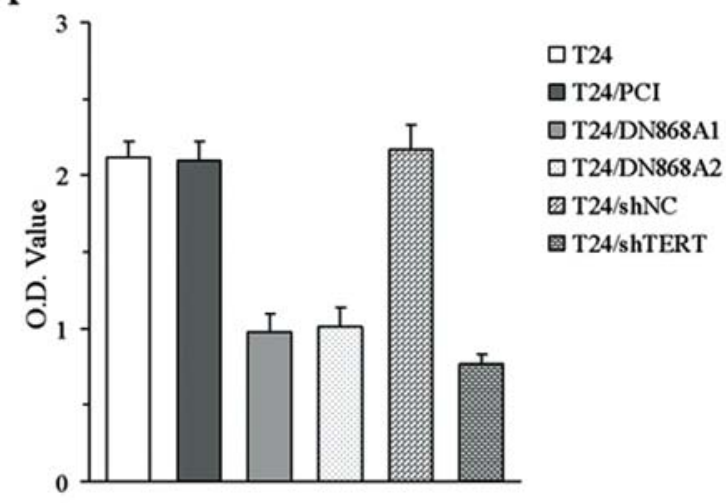

$\mathrm{B}$
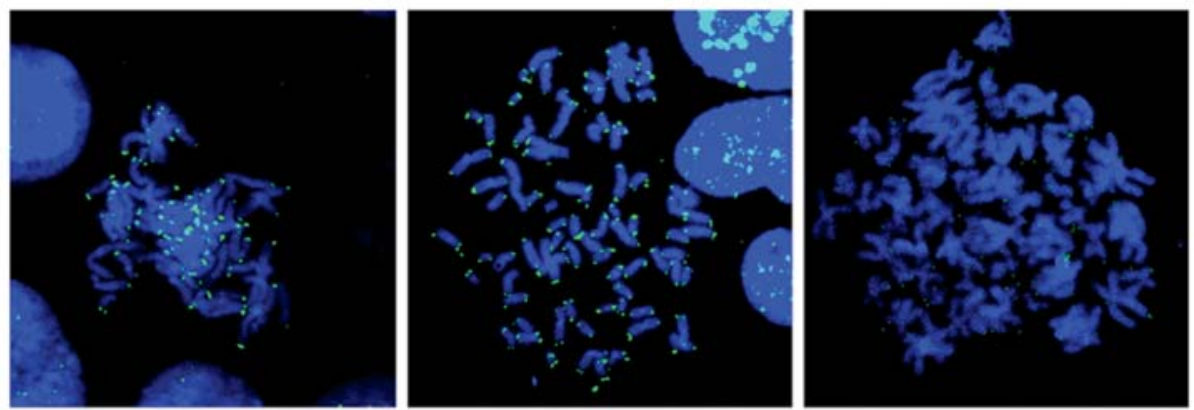

Figure 1. Telomerase activity and telomere length in T24 cells with different transfectants. (A) Telomerase activity in T24 cells transfected with various constructs was determined as described in Materials and methods. Histograms are representative of three independent experiments, while values represent the means of experiments \pm standard errors ('means statistical significance versus the control; p $<0.05$ ). (B) Telomere length analysis of stable cell clone with telomere inhibition by Q-FISH. Individual metaphase chromosomes from T24, T24/PCI and the 8th passage of T24/DN868A subclones were stained using FITC-labeled PNA telomere probe (green) and counterstained with DAPI (blue). The intensity of green signals corresponds to the length of telomeres (magnification, x600).

at most one third at 8th passage comparing with parental or empty vector control T24 cells (Fig. 1A). We next used shRNA to transiently knocked-down hTERT in T24 cell. As shown in Fig. 1A, telomerase activity was significantly shut down when cells treated with shRNA of TERT for $72 \mathrm{~h}$. These results demonstrated that both DN hTERT and shTERT could successfully inhibit telomerase activity.

In addition, we stained the metaphasic chromosomes with a telomeric PNA probe to visualize the phenotype variation of telomere length in T24/DN868A cells. The green signals at the end of chromosome represented the length of telomere. As shown in Fig. 1B, the parental T24 and control T24/PCI cells displayed green signals with similar intensities at the end of almost every chromosome, whereas the 8th passage of T24/DN868A cells exhibited decreased fluorescent signals at the end of chromosome.

Telomerase inhibition suppressed proliferation and tumorigenesis in T24 cells in vitro and in vivo. MTT assay was carried out to determine the subsequent effect of DN hTERT and shRNA on bladder cancer cell growth. As shown in Fig. 2A, cell growth rate was retarded in T24/DN868A cells in a time-dependent manner, moreover, significant growth inhibitory effect was observed at days 5,6 and 7 . Consistently, the growth rate of T24/shTERT cells was dramatically decreased at 3,4 and 5 day after shRNA transfection (Fig. 2B).
Next, we established tumor xenografts by subcutaneous injection of $1 \times 10^{6} \mathrm{~T} 24, \mathrm{~T} 24 / \mathrm{PCI}$, or T24/DN868A cells into 6-8-week old nude mice. The tumor specimens were further analyzed by H\&E staining. We found parental T24 or T24/PCI cells could form detectable tumors as early as 14 days, whereas T24/DN868A cells did not form tumors until 5 weeks after injection. Furthermore, mice injected with T24/DN868A cells demonstrated a sharply decelerated speed in tumor formation (Fig. 2C) with a smaller mean volume of $41.3 \pm 8.9 \mathrm{~mm}^{3}$, whereas the mean tumor volume in mice injected with T24 or T24/PCI cells were $96.3 \pm 12.89$ and $82.6 \pm 10.07 \mathrm{~mm}^{3}$, respectively (Fig. 2D). Histological staining further showed that tumors derived from T24/DN868A cells were less malignant compared to tumors derived from T24 or T24/PCI cells (Fig. 2E).

Cell cycle arrest and apoptosis were induced in T24 cells with telomerase inhibition. To determine the distribution of cells at $\mathrm{G} 1, \mathrm{~S}$ and $\mathrm{G} 2 / \mathrm{M}$ phases after telomerase inhibition, we collected different transfectants and performed flow cytometry analysis. Data showed that the majority of T24/ DN868A and T24/shTERT cells were arrested in G1 phase under telomerase inhibition (G1, 43-49\%; S, 28-30\%), while more parental or control cells entered to the $S$ phase $(\mathrm{G} 1,26-$ 27\%; S, 45-47\%) (Fig. 3A).

In addition, we detected cellular apoptosis in T24 cells with telomerase inhibition by Annexin V-FITC/PI staining. 

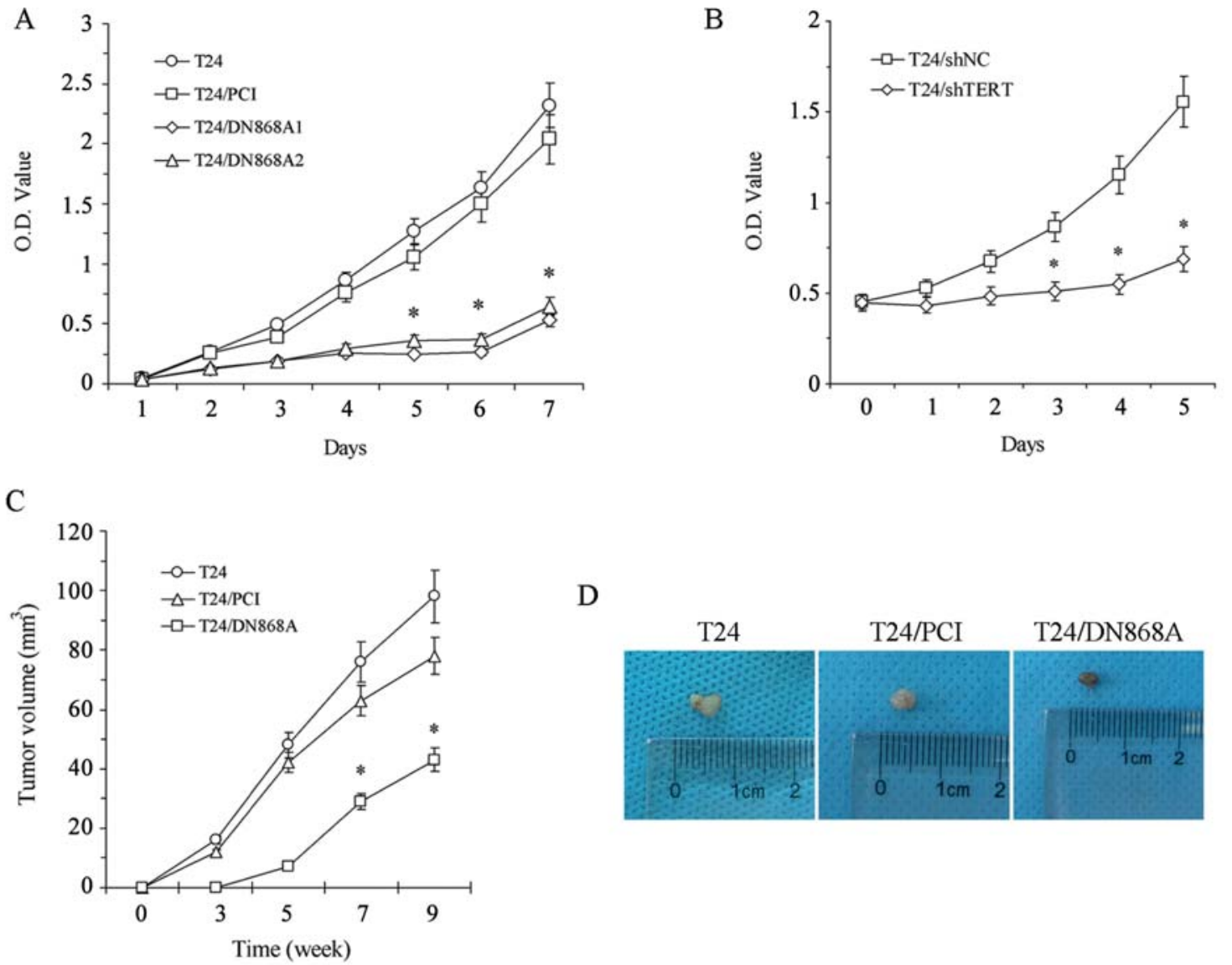

D

E T24

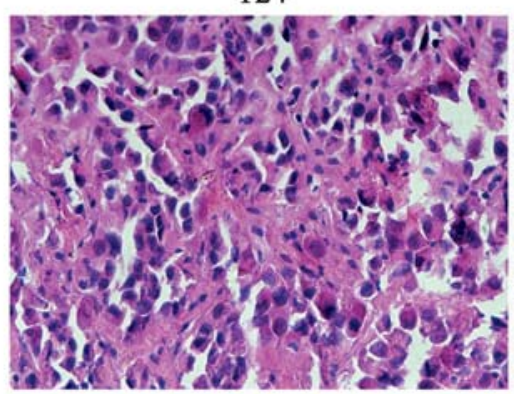

T24/PCI

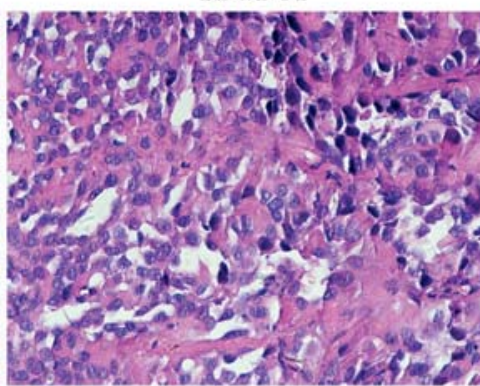

$\mathrm{T} 24$

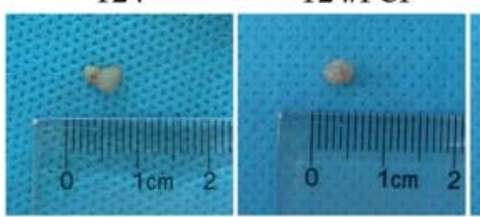

T24/DN868A

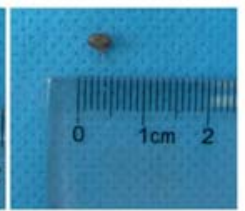

Figure 2. Telomerase inhibition retarded cell growth in bladder cancer. (A and B) MTT assay. Cells with different transfectants were cultured and viability was evaluated as described in Materials and methods. (C and D) Tumorigenicity assay. T24, T24/PCI, and T24/DN868A cells were injected into the abdomen of nude mice, respectively ( $\mathrm{n}=5$ ). After 3 weeks, tumor volume was measured weekly until 8 weeks after injection. (E) HE staining of tumors derived from T24, T24/PCI, and T24/DN868A cells, respectively.

The percentage of apoptotic cells in T24/DN868A and T24/shTERT cells were $14.45 \pm 2.1 \%$ and $18.63 \pm 2.7 \%$ respectively, which were much higher than parental or control cells $(\mathrm{P}<0.05$, Fig. 3B). Since the collapse of mitochondrial transmembrane potential $\left(\Delta \Psi_{\mathrm{m}}\right)$ has been considered critical in triggering apoptotic pathways (17), we assayed the contribution of telomerase inhibition to $\Delta \Psi_{\mathrm{m}}$. Consistently, a significant loss of $\Delta \Psi_{\mathrm{m}}$ was obtained in T24/ DN868A and T24/shTERT cells comparing with parental or vector control cells (Fig. 3C). These data indicate that telomerase inhibition could inhibit cell growth, cause cell cycle arrest at G1 phase and increase cell apoptosis.

PML and p53 were accumulated and colocalized in nucleus after telomerase inhibition. PML has a wide variety of biological functions including induction of apoptosis, senescence, protein turnover, and regulation of gene expression (18). Staining with anti-PML antibody and counterstained with DAPI, we found green PML sparkles were accumulated in the nucleus in T24/DN868A and T24/shTERT cells (Fig. 4). Moreover, Western blotting showed the PML expression was higher in T24/DN868A and T24/shTERT cells, and most of them were located in the nucleus (Fig. 5A and B), and tumor specimens from T24/DN868A cells also showed an overexpression of PML in the nucleus by immunohistochemical staining (Fig. 5C).

p53 is a negative modulator for hTERT, and shares a similar subcellular distribution pattern with hTERT throughout mitosis (19). Here, using double immunofluorescence, we found that more red sparkles of p53 were translocated into 
A

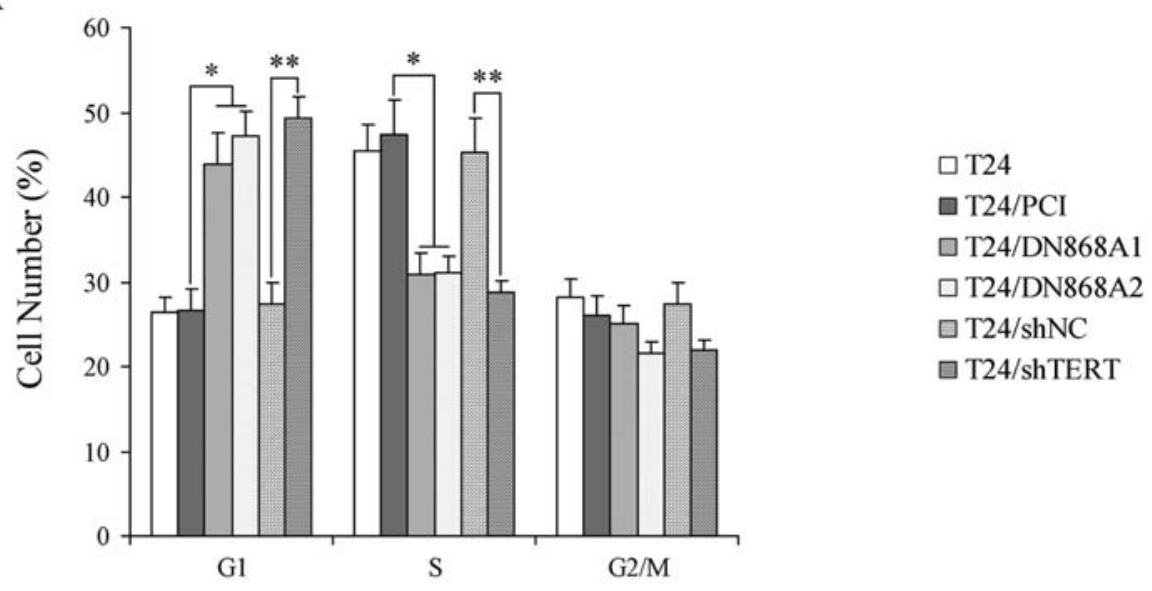

B

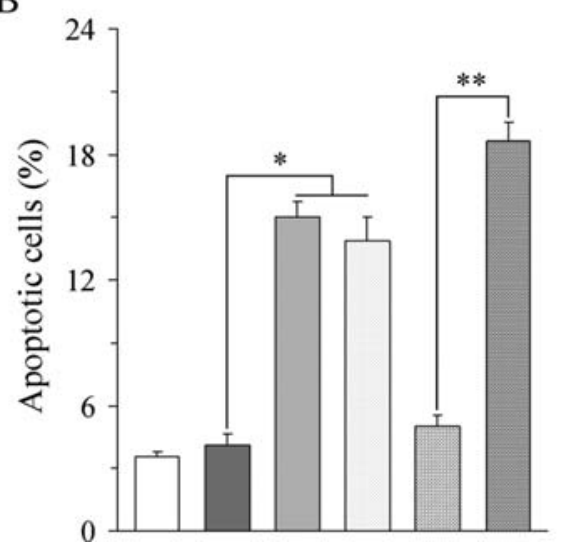

$\mathrm{C}$

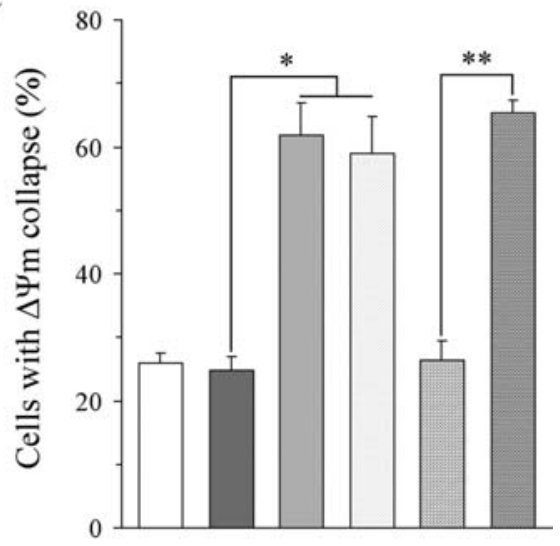

Figure 3. Telomerase inhibition arrested cell cycle and induced apoptosis of bladder cancer cells. (A) Cell cycle assay. (B) Apoptosis assay. (C) Mitochondrial membrane potential $(\Delta \Psi \mathrm{m})$ assay. All experiments were conducted as described in Materials and methods. Histograms were representative of three independent experiments with values representing the means of experiments \pm standard errors ("means significant difference versus the control; $\mathrm{p}<0.05)$.

nucleus in T24/DN868A and T24/shTERT cells comparing with parental or control T24 cells (Fig. 4), and intense p53 nuclear staining was also verified by immunohistochemistry in tumor tissues derived from T24/DN868A cells (Fig. 5C). Notably, most of nuclear p53 spots were colocalized with PML in T24/DN868A and T24/shTERT cells, which were displayed as yellow spots (Fig. 4).

PML was indispensable for p53 activation after telomerase inhibition. PML has previously been shown to regulate p53 localization to PML-NBs in primary or telomerasepositive cells (20). Therefore, siRNA targeting PML was used to determine whether the alteration of p53 resulting from telomerase inhibition was PML-dependent. Immunofluorescence demonstrated that expression of PML was significantly knocked down by siRNA after 72-h transfection (Fig. 6A). Furthermore, we found that most of p53 were displayed in the cytoplasm in PML-knockdown T24/ DN868A cells (Fig. 6B), and one of the p53 downstream target p21 expression was dramatically decreased comparing with control cells (Fig. 6C and D). On the other hand, the enhanced total protein level of p53 after telomerase inhibition was not affected by PML down-regulation (Fig. 6C and D), which indicating that although PML does not interfere with p53 stability or expression, it is indispensable for p53 activation and translocation upon telomerase inhibition.

\section{Discussion}

Activation of telomerase plays an important role in tumor cell immortalization via preventing telomeres from shortening. The enzymatic activity of telomerase is dependent upon hTERT, the catalyst moiety of the telomerase holoenzyme. The rate-limiting step of hTERT in telomerase activation makes it an attractive target for modulating telomerase activity. Numerous reports, which focused on the dysfunction of hTERT by various ways such as mutation in the N-terminus or RNA interference, have demonstrated the obviously impaired effects of telomerase inhibition on cell growth and invasion $(21,22)$. Previous studies have shown that point mutation of the invariant Asp residues (D712, D868, or D869) in the conserved reverse transcriptase regions of hTERT could abolish telomerase activity in the yeast telomerase reverse transcriptase Est2p (23). In the present study, we used a DN hTERT at 868 residues and specific TERT shRNA to inhibit telomerase activity, and then investigated the variation of growth behavior in bladder 


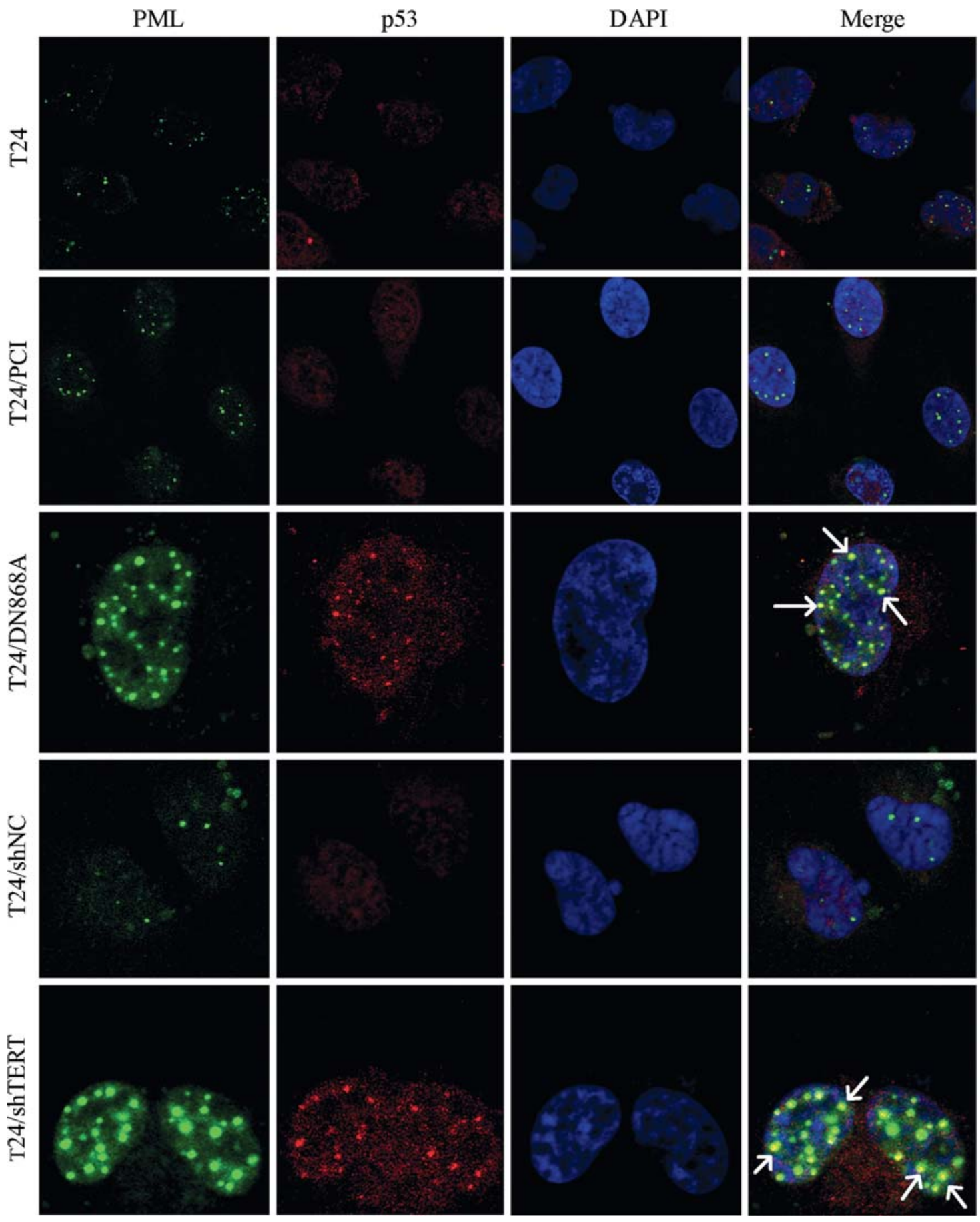

Figure 4. Telomerase inhibition activated PML and p53 nuclear translocation and colocalization. Double immunofluorescence analysis with mouse anti-PML and rabbit anti-p53 in hTERT-inactivated or depleted T24 cells. Green spots represent PML, red spots represent p53, and yellow sparkles indicate the colocalization of PML with p53. PML and p53 were accumulated in nucleus and colocalized with each other in T24/DN868A and T24/shTERT cells (magnification, $\mathrm{x} 600$ ).

cancer. We found both of the DN hTERT and shTERT could successfully inhibit telomerase activity and shorten telomere length. Moreover, telomerase inactivation specially retarded cell growth rate, arrested cell cycle in $\mathrm{G}_{0} / \mathrm{G}_{1}$ phase in vitro and reduced tumorigenicity in vivo. These biological effects are consistent with other mutant hTERT vectors and shRNA $(21,22)$.

The survival of tumor cells depends on the balance between proliferation and apoptosis. In the present study, we explored the apoptotic effects of telomerase inhibition on bladder cancer. Our results displayed a significant increase of apoptosis and dramatic loss of mitochondria membrane potential $\left(\Delta \Psi_{\mathrm{m}}\right)$ in T24/DN868A and T24/shTERT cells. This observation is consistent with recent findings that hTERT possess anti-apoptotic characteristics in many cultured cells $(24,25)$.

Several lines of evidence have demonstrated that PML is a tumor suppressor (8), and it is considered to be an 
A

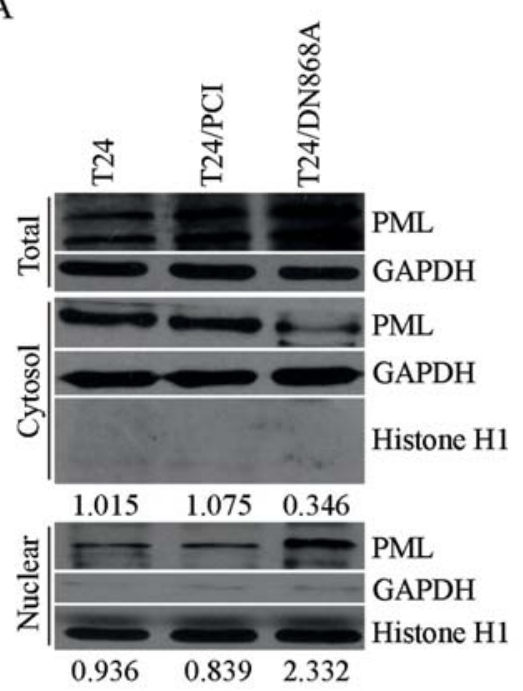

B
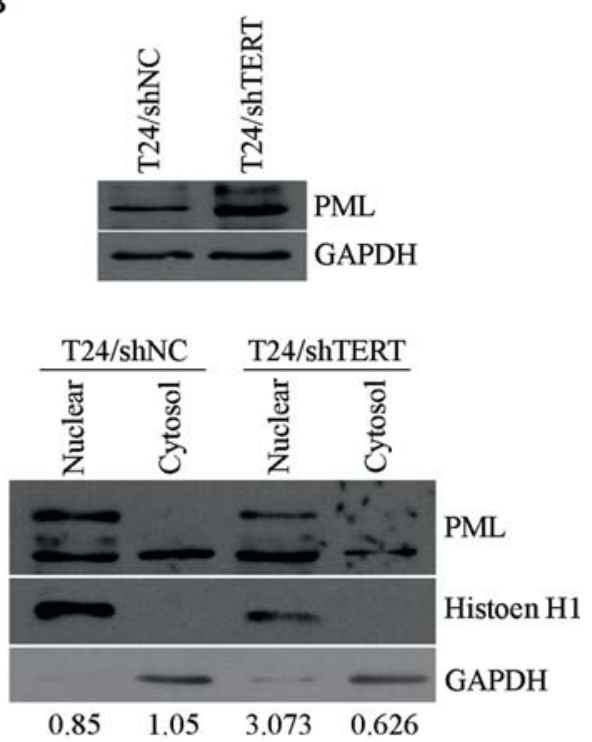

C

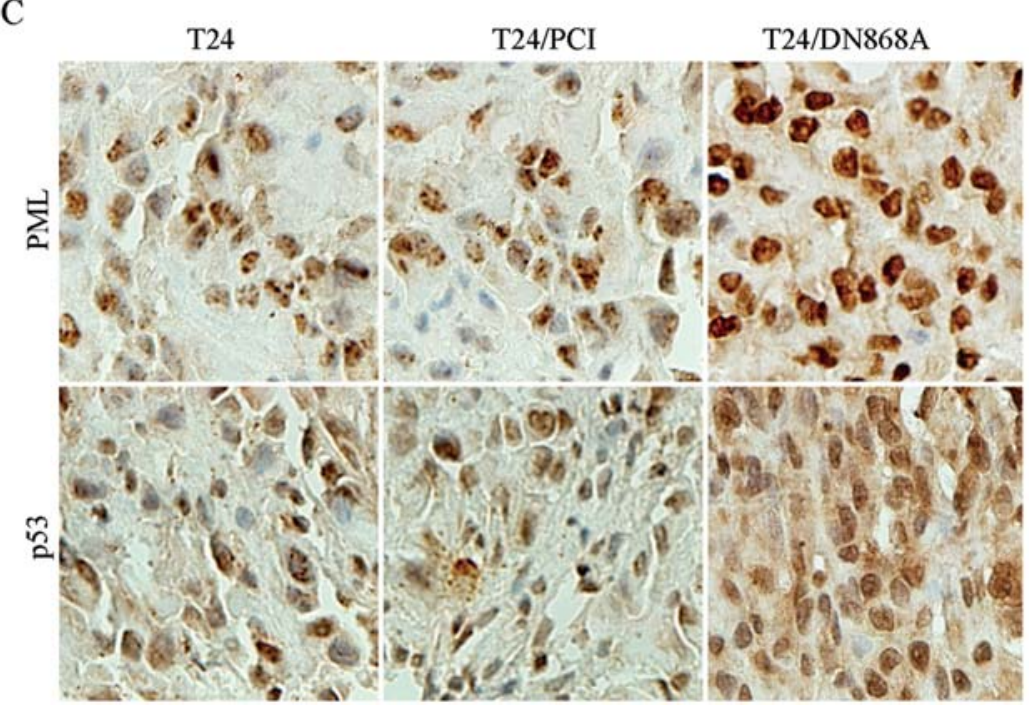

Figure 5. Telomerase inhibition increased PML and p53 expression in vivo and in vitro. (A and B) Total cell lysate, cytosolic fractions and nuclear fractions were prepared and Western blotting was performed to analyze for PML expression. GAPDH and Histone H1 were used as internal control. (C) Immunohistochemical staining of PML and p53 in paraffin-embedded sections from nude mouse tumor samples. Intense staining of nuclear PML and p53 was seen in samples derived from T24/DN868A cells (magnification, $x 600$ ).

important component in the process of cellular senescence and apoptotic responses (26). In cells using a telomeraseindependent mechanism termed alternative lengthening of telomeres (ALT) to maintain telomeres, PML is an indispensable ingredient for the formation of ALT-associated promyelocytic leukemia (PML) nuclear bodies (APBs) (27). In the present study, our results illustrated that PML expression increased and most protein translocated into nucleus from cytoplasm in vitro and in vivo after hTERT inactivation or depletion. Recently, it was reported that PMLIV has a negative effect on the function of telomerase in the post-transcriptional state and possibly induced telomere erosion (28), which supported our speculation from another side. Consistently, loss of PML and increase of hTERT were detected in bladder cancer and associated with tumor invasiveness and malignant grade $(29,30)$. Taken together, it opens up the possibility that dysfunction of TERT and PML may be involved in the onset of urothelial disorders.

p53 is a transcriptional suppressor of hTERT (31), and modulated by PML at multiple levels (32). In this study, enhancement of p53 and p21 expression and accumulation of nucleic p53 foci were observed after telomerase inhibition, and all of these foci shared a similar subcellular distribution pattern with PML. PML-NBs contribute to the post-translational modification of p53 (32), and the C-terminus of PML-IV, which is required for TERT interaction, overlaps with the motif necessary for its interactions with p53 (33). Recent studies have shown that p53-dependent responses are defective in cells lacking expression of the PML gene (34). In the present study, we demonstrated PML was indispensable for p53 translocation and its dependent p21 induction after TERT inactivation, 
A

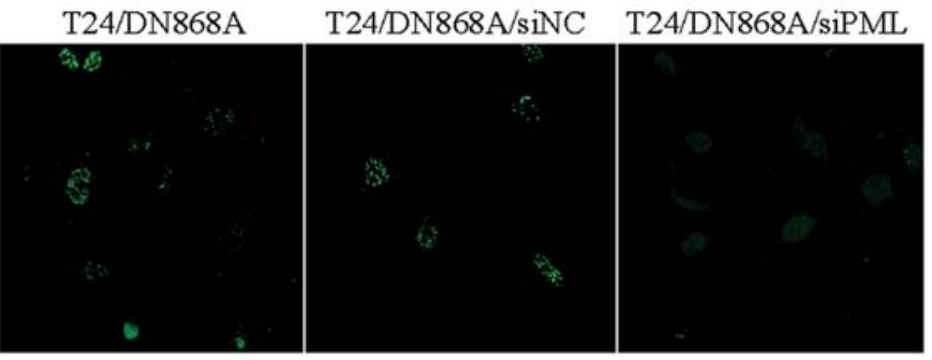

B

$\mathrm{p} 53$

DAPI

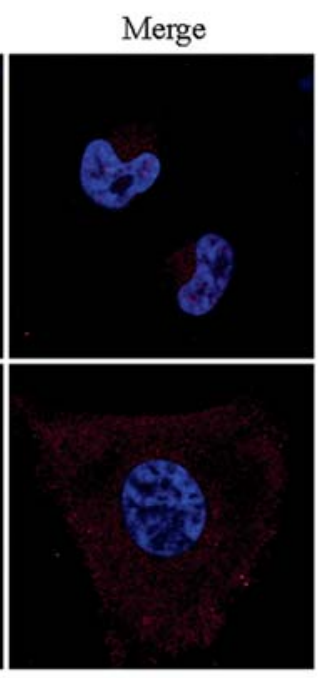

$\mathrm{C}$

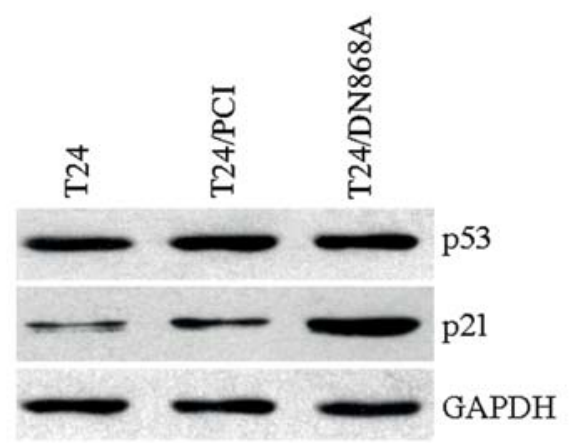

$\mathrm{D}$

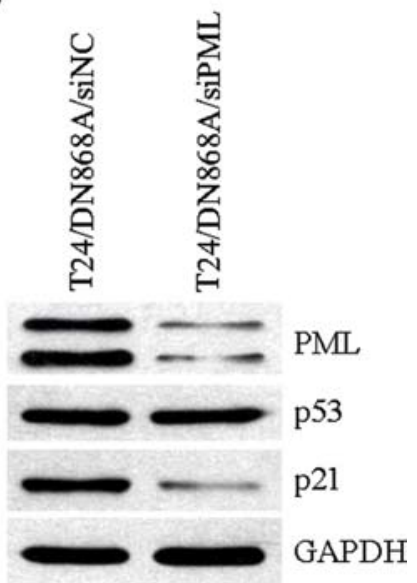

Figure 6. p53 activation upon TERT inactivation relies on PML. (A) Ablation of endogenous PML using siRNA. The level of PML in cells transfected with siPML was subjected to immunofluorescence using anti-PML antibody after $72 \mathrm{~h}$. (B) Immunofluorescence staining of p53 in T24/DN868A cells upon PML depletion. p53 was located in cytoplasm after PML depletion in T24/DN868A cells. (C) Western blotting was performed to analyze for expression of p53 and p21 using relevant antibodies in T24 cells transfected with DN hTERT. GAPDH was used as internal control. Telomerase suppression increased p53 and p21 expression. (D) The TERT-inactivated clone was transfected with PML siRNA for $72 \mathrm{~h}$ and Western blotting was performed to analyze for expression of PML, p53 and p21 using relevant antibodies. GAPDH was used as internal control. PML silencing abrogated p21 induction in TERT-inactivated T24 cells.

while it does not affect the total p53 protein levels. This result suggests PML may mediate the interaction between TERT and other PML-associating enzymes, such as p53.

In summary, our data show that growth inhibition associated with telomerase suppression could be induced by a point mutation vector of hTERT at 868 residues or shRNA. Translocation of PML and p53 and their colocalization in bladder cancer T24 cells suggests that the growth arrest and apoptosis caused by telomerase inhibition is mediated by PML.

\section{Acknowledgements}

This study was supported by the program of The National Natural Science Foundation of China (NSFC No. 30772163). We thank Drs Junping Liu (Monash University Medical 
School, Australia) for providing DN hTERT plasmid and Leland W.K. Chung (Emory University School of Medicine, Atlanta, GA, USA) for providing T24 cell line.

\section{References}

1. Razak ZR, Varkonyi RJ, Kulp-McEliece M, Caslini C, Testa JR, Murphy ME and Broccoli D: p53 differentially inhibits cell growth depending on the mechanism of telomere maintenance. Mol Cell Biol 24: 5967-5977, 2004.

2. Kim NW, Piatyszek MA, Prowse KR, et al: Specific association of human telomerase activity with immortal cells and cancer. Science 266: 2011-2015, 1994.

3. McEachern MJ, Krauskopf A and Blackburn EH: Telomeres and their control. Annu Rev Genet 34: 331-358, 2000.

4. Nakayama J, Tahara H, Tahara E, et al: Telomerase activation by hTRT in human normal fibroblasts and hepatocellular carcinomas. Nat Genet 18: 65-68, 1998.

5. Zhang X, Mar V, Zhou W, Harrington L and Robinson MO: Telomere shortening and apoptosis in telomerase-inhibited human tumor cells. Genes Dev 15: 2388-2399, 1999.

6. Chang S and DePinho RA: Telomerase extracurricular activities. Proc Nati Acad Sci USA 99: 12520-12522, 2002.

7. Melnick A and Licht JD: Deconstructing a disease: RARalpha, its function partners, and their roles in the pathogenesis of acute promyelocytic leukemia. Blood 93: 3167-3215, 1999.

8. Bernardi R and Pandolfi PP: Structures, dynamics and functions of promyelocytic leukaemia nuclear bodies. Nat Rev Mol Cell Biol 8: 1006-1016, 2007.

9. Zhong S, Salomoni P and Pandolfi PP: The transcriptional role of PML and the nuclear body. Nat Cell Biol 2: E85-E90, 2000.

10. He DL, Zhu AJ and Wang HY: Expression of PML growth suppressor in bladder cancer. Natl Med J Chin 81: 881-882, 2001

11. Li L, He DL, He H, Wang XY, Zhang LL, Luo Y and Nan XY: Overexpression of PML induced apoptosis in bladder cancer cell by caspase dependent pathway. Cancer Lett 236: 259-268, 2006.

12. Borden KL: Pondering the promyelocytic leukemia protein (PML) puzzle: possible functions for PML nuclear bodies. Mol Cell Biol 22: 5259-5269, 2002.

13. Lansdorp PM, Verwoerd NP, Van de Rijke FM, et al: Heterogeneity in telomere length of human chromosomes. Hum Mol Genet 5: 685-691, 1996.

14. Li L, Zhang D, Zhang LL, et al: PrlZ expression is associated with the progression of prostate cancer LNCaP cells. Mol Carcinog 48: 432-440, 2009.

15. Zhu G, Zhau HE, He H, et al: Sonic and desert hedgehog signaling in human fetal prostate development. Prostate 67: 674-684, 2007

16. Santiago AR, Cristovao AJ, Santos PF, Carvalho CM and Ambrosio AF: High glucose induces caspase-independent cell death in retinal neural cells. Neurobiol Dis 25: 464-472, 2007.
17. Ledgerwood EC and Morison IM: Targeting the apoptosome for cancer therapy. Clin Cancer Res 15: 420-424, 2009.

18. Shen TH, Lin HK, Scaglioni PP, Yung TM and Pandolfi PP: The mechanisms of PML-nuclear body formation. Mol Cell 24: 331-339, 2006.

19. Xu D, Wang Q, Gruber A, et al: Downregulation of telomerase reverse transcriptase mRNA expression by wild type p53 in human tumor cells. Oncogene 19: 5123-5133, 2000.

20. Salomoni P and Pandolfi PP: The role of PML in tumor suppression. Cell 108: 165-170, 2002.

21. Kraemer K, Fuessel S, Schmidt U, Kotzsch M, Schwenzer B, Wirth MP and Meye A: Antisense-mediated hTERT inhibition specifically reduces the growth of human bladder cancer cells. Clin Cancer Res 9: 3794-3800, 2003.

22. Zhang P, Chen Y, Jiang X, Tu Z and Zou L: Tumor-targeted efficiency of shRNA vector harboring chimera hTERT/U6 promoter. Oncol Rep 23: 1309-1316, 2010.

23. Liu JP: Studies of the molecular mechanisms in the regulation of telomerase activity. FASEB J 13: 2091-2104, 1999.

24. Zhu H, Fu W and Mattson MP: The catalytic subunit of telomerase protects neurons against amyloid beta-peptideinduced apoptosis. J Neurochem 75: 117-124, 2000.

25. Cao Y, Li H, Mu FT, Ebisui O, Funder JW and Liu JP: Telomerase activation causes vascular smooth muscle cell proliferation in genetic hypertension. FASEB J 16: 1096-1098, 2002.

26. Bernardi R and Pandolfi PP: Role of PML and the PML-nuclear body in the control of programmed cell death. Oncogene 22: 9048-9057, 2003

27. Jiang WQ, Zhong ZH, Henson JD and Reddel RR: Identification of candidate alternative lengthening of telomeres genes by methionine restriction and RNA interference. Oncogene 26: 4635-4647, 2007

28. Oh W, Ghim J, Lee EW, Yang MR, Kim ET, Ahn JH and Song J: PML-IV functions as a negative regulator of telomerase by interacting with TERT. J Cell Sci 122: 2613-2622, 2009.

29. Gurrieri C, Capodieci P, Bernardi R, et al: Loss of the tumor suppressor PML in human cancers of multiple histologic origins. J Nati Cancer Inst 96: 269-279, 2004.

30. Yang $\mathrm{CH}$, Hung WC, Wang SL, et al: Immunoexpression and prognostic role of hTERT and cyclin D1 in urothelial carcinoma. APMIS 116: 309-316, 2008.

31. Li H, Cao Y, Berndet MC, Funder JW and Liu JP: Molecular interactions between telomerase and the tumor suppressor protein p53 in vitro. Oncogene 18: 6785-6794, 1999.

32. Pearson M, Carbone R, Sebastiani C, et al: PML regulates p53 acetylation and premature senescence induced by oncogenic Ras. Nature 406: 207-210, 2000

33. Fogal V, Gostissa M, Sandy P, et al: Regulation of p53 activity in nuclear bodies by a specific PML isoform. EMBO J 19: 6185-6195, 2000

34. Guo A, Salomoni P, Luo J, Shin A, Zhong S, Gu W and Pandolfi PP: The funtion of PML in p53-dependent apoptosis. Nat Cell Biol 2: 730-736, 2000. 